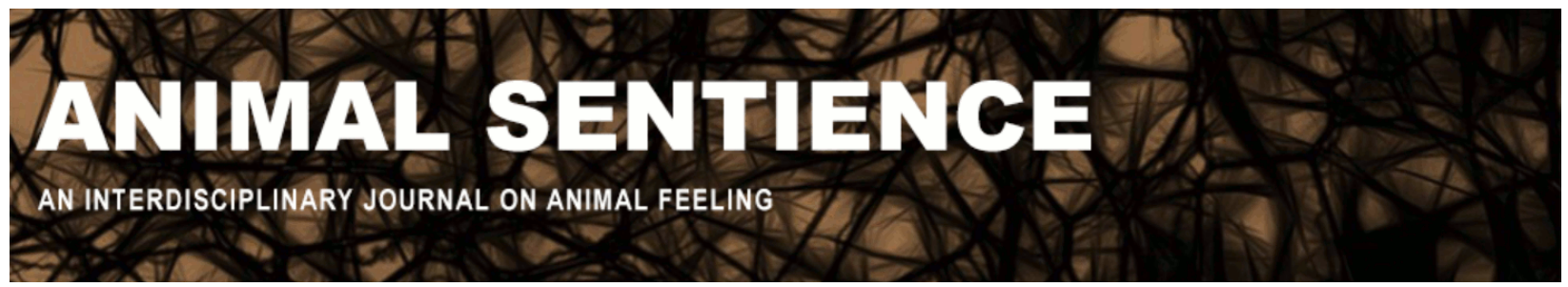

Boch, Magdalena and Lamm, Claus (2017) The multiple facets of empathy. Animal Sentience 14(14)

DOI: $10.51291 / 2377-7478.1278$

Date of submission: 2017-12-18

Date of acceptance: 2017-12-22

(c) 


\title{
The multiple facets of empathy
}

\author{
Commentary on Kujala on Canine Emotions
}

\section{Magdalena Boch and Claus Lamm \\ Social, Cognitive and Affective Neuroscience Unit \\ Department of Basic Psychological Research and Research Methods \\ University of Vienna, Austria}

\begin{abstract}
We discuss the definition of empathy provided by Kujala (2017) and argue that research in this field, in assigning the cognitive component of empathy only a secondary role, misses crucial information. Further knowledge about dogs' ability for higher cognitive processes helps (a) in interpreting results such as potential prosocial behavior in dogs and (b) sheds light on the question of whether abilities like perspective-taking and self-other distinction are uniquely human.
\end{abstract}

Magdalena Boch is a PhD student at the University of Vienna, Austria. Her research interests include social and comparative cognition with a focus on the evolutionary roots of the social brain by comparing the neural underpinnings of social skills in dogs and humans. scan.psy.univie.ac.at

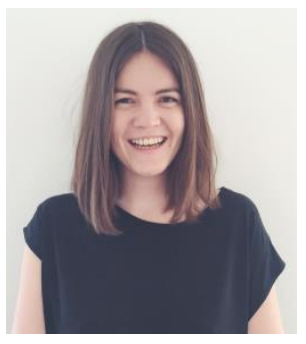

Claus Lamm is a Professor of Biological Psychology and head of the Social, Cognitive and Affective Neuroscience (SCAN) Unit at the University of Vienna, Austria. His research interests include the underlying psychological, biological and behavioral mechanisms of social cognition, with a main interest in the proximate and ultimate mechanisms of empathy.

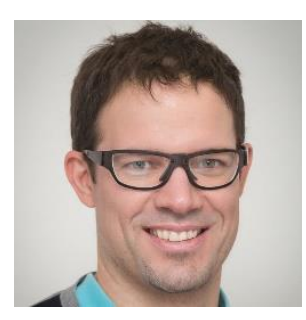
homepage.univie.ac.at/claus.lamm

Empathy is a cornerstone of social interaction and a crucial component of emotional experience in humans. Providing a more decisive answer as to whether empathy should be considered a uniquely human skill is hindered by a lack of conceptual clarity, universally accepted definitions, and a sense of what processes constitute empathy (cf. Batson, 2009; Bernhardt \& Singer, 2012). This is true in social psychological and neuroscience research on humans, but also in comparative and non-human animal research. Definitions of empathy in non-human animal research are often limited to automatic, affective, bottom-up processes such as emotional contagion (PérezManrique \& Gomila, 2017; Silva \& de Sousa, 2011), whereas in humans empathy is often defined as a multi-faceted phenomenon derived from the interplay of bottom-up and top-down processes (Decety \& Jackson, 2004), including a host of cognitive processes. In her target article, Kujala (2017) provides a well-balanced and substantive review of social cognition in general and 
specifically empathic-like responses and behaviors in dogs, pointing out the need for future research on related topics in cognition, such as theory of mind in dogs. We would like to complement her efforts, putting further emphasis on the importance of additional cognitive components of empathy in order to better understand whether dogs experience "full-blown" empathy or only precursors of it.

\section{Defining empathy and its constituents}

Before making our point on how cognitive considerations can add to our understanding of the affective components of empathy, we would like to briefly recap the definition(s) of empathy in the human literature.

\subsection{Affect sharing and the self-other distinction}

The ability to share another individual's feeling is certainly crucial for empathy. However, most models of empathy, at least in the human literature, stress that affect sharing is only one component. It is also important that the individual who is sharing the other's affect is able to distinguish between self and other, that is, that they know that the other person is the source of their own affective state (De Vignemont \& Singer, 2006; Yamamoto, 2017). This leads to the question of whether cognitive or affective processes alone can generate a multi-faceted phenomenon such as empathy or whether it is their interplay that generates the full-blown experience of empathy. In other words, can someone, in our case, a dog, experience empathy and as a result act to help or comfort another individual without having (some form of) awareness of the difference between self and other?

\subsection{Empathy and emotional contagion}

To address this question, the concept of emotional contagion must be disentangled from empathy. Kujala divides emotional empathy into either emotional-contagion/self-distress or empathic concern. This accords with the empathy model of de Waal \& Preston (2017), with emotional contagion as an automatic affective response matching the affective state of another individual (see also Preston \& de Waal, 2001). We (Singer \& Lamm, 2009) have suggested a distinction between emotional contagion and empathy, the latter comprised of bottom-up and top-down processes such as cognitive appraisal instead of only simple automatic processes (Lamm \& Majdandžić, 2015). Emotional contagion lacks the self-other distinction. It can thus lead to personal distress because there is no top-down process to correctly assign the affective response (to the other) or to regulate it. However, both phenomena, emotional contagion and empathy, are equally interesting for comparative research. To better understand their relationship, they should be categorized and investigated as distinct phenomena in a process model. Despite the advantages of self-report and introspection offered by human research, differentiating between empathy and other related phenomena is still very challenging even in humans (Batson, 2009; Lamm, Rütgen, \& Wagner, 2017). It is hence expected that it is even more difficult with dogs. 


\section{Why cognitive aspects of empathy matter}

The question we raised in the beginning was: "Can someone, in our case, a dog, experience empathy and as a result act to help or comfort another individual without having (some form of) awareness of the difference between self and other?" Our tentative answer would be that empathy-like behavior can be observed whether or not the individual has self-awareness but the underlying processes and motives are different. Kujala addresses the difficulty of differentiating between actual prosocial or empathic responses and behavior aimed at stress reduction. The former could result from an interplay of both top-down and bottom-up processes, whereas the latter might originate from a behavioral response to automatic affective processes that are "selfishly motivated." (This has been extensively shown in social psychological and neuroscientific research attempting to determine whether prosocial behavior is "truly" altruistic, or just results from the selfish motive to reduce one's own distress; Batson, 2011; Lamm et al., 2017; Lamm, Batson, \& Decety, 2007). Investigating the cognitive abilities of dogs would enable us to move from automatic bottom-up processes to higher-level cognition, clarifying which components or processes of empathy (as defined in humans) dogs might also possess (and whether, or to what extent, empathy is uniquely human). More knowledge about the processes underlying empathylike responses can help us move beyond anthropomorphic projections. Weiss, Inoue-Murayama, King, Adams, \& Matsuzawa (2012), for example, describe how an anthropomorphic interpretation does not need to be the only one in personality research on non-human primates.

Expanding the definition of empathy in non-human animal research from affective to cognitive processes comes with several advantages. First, as argued in the previous section, the ability to take another individual's perspective is the crucial difference between simply catching another individual's affective state and being aware of and understanding their emotional state. This can shed new light on the motives underlying empathy-like behavior in dogs and the function of their affective abilities, which in turn leads to the question of whether higher cognitive processes are solely human or the existence of similar abilities in dogs might be the reason for the development of the unique relationship between dogs and humans.

A shared understanding of empathy across research traditions, sub-disciplines and approaches will also facilitate comparative research. We accordingly second Kujala's call for more research in the fields studying theory of mind and related concepts such as perspective-taking but would add that the self-other distinction as well as self- and other-related motives seem even more crucial.

\section{References}

Batson, C. D. (2009). These things called empathy: Eight related but distinct phenomena. In J. Decety \& W. Ickes (Eds.), The Social Neuroscience of Empathy (pp. 3-16). Cambridge, MA: MIT Press.

Batson, C. D. (2011). Altruism in Humans. New York, NY: Oxford University Press, Inc. Bernhardt, B. C., \& Singer, T. (2012). The Neural Basis of Empathy. Annual Review of Neuroscience, 35(1), 1-23.

de Waal, F. B. M., \& Preston, S. D. (2017). Mammalian empathy: Behavioural manifestations and 
neural basis. Nature Reviews Neuroscience, 18(8), 498-509.

Decety, J., \& Jackson, P. L. (2004). The functional architecture of human empathy. Behavioral and Cognitive Neuroscience Reviews, 3(2), 71-100.

Kujala, M. (2017). Canine emotions as seen through human social cognition. Animal Sentience 14(1).

Lamm, C., \& Majdandžić, J. (2015). The role of shared neural activations, mirror neurons, and morality in empathy - A critical comment. Neuroscience Research, 90, 15-24.

Lamm, C., Batson, C. D., \& Decety, J. (2007). The neural substrate of human empathy: Effects of perspective-taking and cognitive appraisal. Journal of Cognitive Neuroscience, 19(1), 42-58.

Lamm, C., Rütgen, M., \& Wagner, I. C. (2017). Imaging empathy and prosocial emotions. Neuroscience Letters, EPub ahead of print.

Pérez-Manrique, A., \& Gomila, A. (2017). The comparative study of empathy: Sympathetic concern and empathic perspective-taking in non-human animals. Biological Reviews.

Preston, S. D., \& de Waal, F. B. M. (2001). Empathy: Its ultimate and proximate bases. Behavioral and Brain Sciences, 25(1), 1-20.

Silva, K., \& de Sousa, L. (2011). 'Canis empathicus'? A proposal on dogs' capacity to empathize with humans. Biology Letters, 7(4), 489-492.

Singer, T., \& Lamm, C. (2009). The social neuroscience of empathy. Annals of the New York Academy of Sciences, 1156, 81-96.

Weiss, A., Inoue-Murayama, M., King, J. E., Adams, M. J., \& Matsuzawa, T. (2012). All too human? Chimpanzee and orang-utan personalities are not anthropomorphic projections. Animal Behaviour, 83(6), 1355-1365.

Yamamoto, S. (2017). Primate empathy: Three factors and their combinations for empathyrelated phenomena. Wiley Interdisciplinary Reviews: Cognitive Science, 8(3), e1431. 